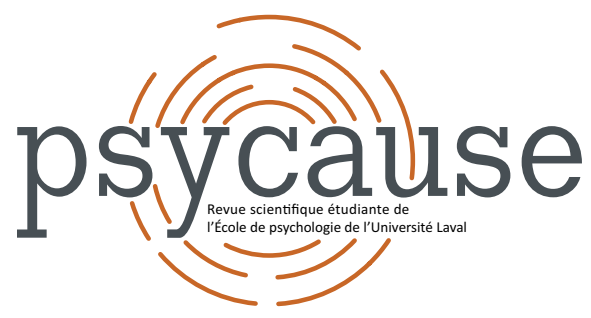

\title{
PSYCAUSE
}

Revue scientifique étudiante de l'École de psychologie de l'Université Laval

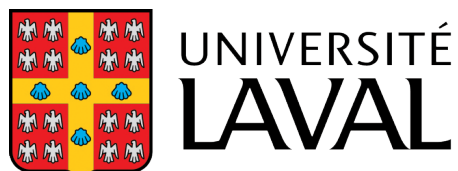

Faculté des sciences sociales École de psychologie

\section{ÉDITORIAL - L'IMPACT DE LA PANDÉMIE DE MALADIE À CORONAVIRUS (COVID-19) SUR LA RÉDACTION ET LA PUBLICATION D'ÉCRITS SCIENTIFIQUES}

David PAQUET ${ }^{1 *}$, Frédéric THÉRIAULT-COUTURE ${ }^{*}$

1 École de psychologie, Université Laval, Québec, QC, Canada

*revuepsycause@psy.ulaval.ca

\section{Pour citer l'article}

Paquet, D., \& Thériault-Couture, F. (2021). Éditorial - L'impact de la pandémie de maladie à coronavirus (COVID-19) sur la rédaction et la publication d'écrits scientifiques. Psycause: Revue scientifique étudiante de l'École de psychologie de l'Université Laval, 11(1), 3.

\section{Droits d'auteur}

(C) 2021 Paquet \& Thériault-Couture. Cet article est distribué en libre accès selon les termes d'une licence Creative Commons Attribution 4.0 International (de type CC-BY 4.0) qui permet l'utilisation du contenu des articles publiés de façon libre, tant que chaque auteur ou autrice du document original à la publication de l'article soit cité(e) et référencé(e) de façon appropriée. 


\section{ÉDITORIAL - L'IMPACT DE LA PANDÉMIE DE MALADIE À CORONAVIRUS (COVID-19) SUR LA RÉDACTION ET LA PUBLICATION D'ÉCRITS SCIENTIFIQUES}

David Paquet ${ }^{1 *}$, Frédéric Thériault-Couture ${ }^{1 *}$

1 École de psychologie, Université Laval, Québec, QC, Canada

*revuepsycause@psy.ulaval.ca

Depuis le mois de mars 2020, la maladie à coronavirus (COVID-19) a porté atteinte à l'ensemble des sphères de la société, que ce soit au niveau de l'économie, de l'éducation, de la santé, ou encore des contacts sociaux. C'est un événement sans précédent dans l'histoire récente, qui engendrera de multiples conséquences à court, moyen et long terme. Le domaine de la recherche scientifique souffrira-t-il de ces répercussions? La recherche souffrirait-elle déjà des impacts de la pandémie?

La littérature indique que le domaine scientifique ressent déjà l'effet de ces restrictions. Il devient plus complexe de mener des expériences en laboratoire, étant donné que les données de travaux déjà en cours ont été perdues ou suspendus et que plusieurs chercheur.es et étudiant.es ont rapporté une diminution de leur productivité ainsi qu'un plus grand sentiment de stress et d'anxiété face au fait de mener leurs projets à terme (Korbel et Stegle, 2020). Cette suspension des travaux de recherche peut ainsi avoir un impact sur le nombre d'articles scientifiques subséquemment publiés (Korbel et Stegle, 2020). II est cependant pertinent de constater aussi certains effets positifs de la pandémie, comme l'aspect convivial du travail à la maison, qui diminue les allers-retours au travail. La communication à distance a été d'autant plus facilitée, grâce à la montée en popularité des applications comme Zoom ou Microsoft Teams, qui facilitent l'établissement de réunions d'équipe en virtuel (Serhan, 2020).

Le nombre d'articles scientifiques inclus dans la présente édition de la revue Psycause, plus faible que les années précédentes, pourrait possiblement refléter une forme de ralentissement de la recherche scientifique étudiante au courant de la pandémie de la COVID-19. Bien que l'édition soit moins étoffée en ce début d'année 2021, la qualité des articles qui y sont rédigés $n^{\prime}$ en est pas moindre. Du traitement des joueurs pathologiques aux conséquences des pensionnats autochtones, en passant par les saines habitudes de vie à l'aide du numérique, la publication de ces trois articles est un témoignage de la persistance de la rédaction scientifique étudiante, et ce, peu importe le contexte dans lequel nous vivons. Pandémie ou non, la revue Psycause se consacre à encourager et à favoriser la rédaction, la publication et la communication d'articles scientifiques étudiants. En ce sens, nous travaillons déjà sur notre prochaine édition à paraître au début de l'automne. Celle-ci portera sur les résumés longs de travaux étudiant menés dans le cadre des cours Recherche Dirigée I et II au baccalauréat en psychologie.

Enfin, pour toutes suggestions nous permettant d'améliorer la revue ou de nous rapprocher de notre objectif, nous vous invitons à écrire à l'adresse suivante: revuepsycause@psy.ulaval.ca

Bonne lecture,

Frédéric Thériault-Couture, éditeur en chef

David Paquet, éditeur en chef adjoint

\section{Références}

Korbel, J. O., \& Stegle, O. (2020). Effects of the COVID-19 pandemic on life scientists. Genome Biology, 21, 112. https:// doi.org/10.1186/s13059-020-02031-1

Serhan, D. (2020). Transitioning from Face-to-Face to Remote Learning: Students' Attitudes and Perceptions of Using Zoom during COVID-19 Pandemic. International Journal of Technology in Education and Science, 4(4), 335-342. https://doi.org/10.46328/ijtes.v4i4.148

\section{Pour citer l'article}

Paquet, D., \& Thériault-Couture, F. (2021). Éditorial - L'impact de la pandémie de maladie à coronavirus (COVID-19) sur la rédaction et la publication d'écrits scientifiques. Psycause: Revue scientifique étudiante de l'École de psychologie de I'Université Laval, 11(1), 3. 


\section{Droits d'auteur}

(C) 2021 Paquet \& Thériault-Couture. Cet article est distribué en libre accès selon les termes d'une licence Creative Commons Attribution 4.0 International (de type CC-BY 4.0) qui permet l'utilisation du contenu des articles publiés de façon libre, tant que chaque auteur ou autrice du document original à la publication de l'article soit cité(e) et référencé(e) de façon appropriée.

\section{OBJECTIFS ET MODE DE FONCTIONNEMENT DE LA REVUE}

La création de la revue dans sa forme actuelle vise les objectifs suivants:

(1). Informer et initier les étudiant(e)s de premier cycle au processus de révision par les pairs. Notre objectif premier est donc d'initier les étudiant(e)s au monde de l'écriture/de la révision scientifique.

(2). Vulgariser et diffuser les travaux et projets de recherche des étudiant(e)s du baccalauréat, de la maîtrise et du doctorat à l'ensemble de l'École de psychologie, mais aussi à la communauté universitaire.
Pour la publication de ces articles, le journal s'est doté d'une structure de révision par les pairs. Le processus de publication et de révision a été appliqué pour la première fois d'octobre 2016 à juin 2017. Toute idée ou proposition quant au processus de révision est la bienvenue! Vous pouvez nous écrire à revuepsycause@psy.ulaval.ca.

\section{Soumission de résumés préliminaires} Commentaires sur les résumés
Réception des articles
Révision des articles

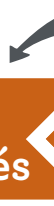
par un prof

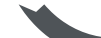

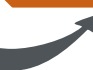

Corrections de français 